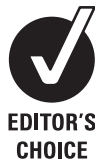

CHOICE

\title{
Epidemiology and treatment outcome of childhood tuberculosis in England and Wales: 1999-2006
}

\author{
I Abubakar, ${ }^{1,2}$ M T Laundy, ${ }^{1}$ C E French, ${ }^{1}$ D Shingadia ${ }^{3}$
}

${ }^{1}$ Health Protection Agency, Centre for Infections, London, UK; ${ }^{2}$ School of Medicine, Health Policy and Practice, University of East Anglia, Norwich, UK; ${ }^{3}$ Great Ormond Street Hospital for Children, London, UK

Correspondence to: Ibrahim Abubakar, Tuberculosis Section, Respiratory Diseases Department, Health Protection Agency, Centre for Infections,

61 Colindale Avenue,

London NW9 5EQ, UK:

ibrahim.abubakar@hpa.org.uk

Accepted 6 June 2008 Published Online First

18 June 2008

\begin{abstract}
Objective: To describe the recent trends in demographic, clinical and microbiological characteristics and outcome of treatment in paediatric cases of tuberculosis.

Design: National surveillance study.

Setting: England and Wales.

Patients: All children under the age of 16 years reported with tuberculosis to the national enhanced surveillance system between 1999 and 2006 were included.
\end{abstract}

Main outcome measures: Proportions, and rates of disease, by demographic characteristics, site of disease, diagnostic delay, culture confirmation, species, drug susceptibility and treatment outcome.

Results: 3563 cases of tuberculosis in children were reported between 1999 and 2006 . The incidence rate remained stable at around 4.3 per $100000(95 \% \mathrm{Cl} 4.1$ to 4.4). Patients born outside the UK had a tuberculosis rate higher than children born in the UK (37 per 100000 vs 2.5 per 100000 ) and this rate increased over the period. Rates in the black African ethnic group were highest at 88 per $100000.60 \%$ of children had pulmonary disease, the commonest presentation, but only $948(27 \%)$ had culture confirmed tuberculosis. The median time to diagnosis from onset of symptoms was 37 days (interquartile range 12-89). The proportions of cases with rifampicin, isoniazid and multi-drug resistant isolates were $2.4 \%, 9.3 \%$ and $2.3 \%$, respectively. $88 \%$ of children completed treatment and less than $1 \%$ died.

Conclusions: Overall rates of tuberculosis in children have remained stable, with the majority completing treatment. Rates are, however, highest in children not born in the UK, particularly among certain ethnic minority groups. Levels of drug resistance are also high.

Although nearly $11 \%$ of all tuberculosis cases globally occur in children less than 15 years of age, ${ }^{1}$ childhood tuberculosis is not prioritised by national control programmes because children contribute little to transmission. The World Health Organization (WHO) has recently published guidelines for national programmes for the diagnosis and management of childhood tuberculosis. ${ }^{2}{ }^{3}$ These recommendations aim to improve the surveillance, management and control of childhood tuberculosis.

Tuberculosis rates have been rising in England and Wales since 1987. ${ }^{4}$ The most recent report showed an incidence of 15 per 100000 in 2006. Despite this increase, the most recent study of epidemiology in children was based on the 1998 survey. ${ }^{5}$ The survey showed significant changes in the epidemiology of tuberculosis over a decade, but the overall incidence of disease was stable. A more recent review of childhood tuberculosis in Wales showed that incidence remained low, ${ }^{6}$ although

\section{What is already known on this topic}

- The overall rate of tuberculosis in the general population in England and Wales is increasing.

- The distribution of disease continues to disproportionately affect children of certain ethnic groups.

\section{What this study adds}

- Rates of tuberculosis in children remain low in England and Wales and are not increasing.

- Few cases in children are reported to have microbiological confirmation and drug resistance rates are higher than in the adult population.

- The proportion of children completing treatment is consistently high and the mortality rates are low.

rates are increasing among black Africans. Monitoring tuberculosis rates in children is important because rates reflects recent transmission within the community and, therefore, serve as a good measure of overall tuberculosis control.

This study describes recent trends in the demographic, clinical and microbiological characteristics of tuberculosis cases in children in England and Wales, and examines the outcome of treatment.

\section{METHODS}

\section{Data sources}

All cases ( $<16$ years of age) reported to the Enhanced Tuberculosis Surveillance (ETS) system by clinical teams in England and Wales between 1999 and 2006 were included in the study. Data collected through this system include notification details and demographic and clinical information.

Further microbiological information on Mycobacterium tuberculosis complex isolates was obtained from a network of five mycobacterial reference laboratories. These data were linked to the ETS dataset using in-house computer software which produced pairs of possible matches based on names, sex, residential address and date of birth. Pairs with a very high degree of similarity were automatically matched. Further pairs with a high matching score were reviewed individually by comparing the patient identifiable information and additional matches were obtained. Pairs with a low score were automatically excluded. Microbiological data collected through this network included species ( $M$ tuberculosis, $M$ bovis or 
$M$ africanum) and drug susceptibility to rifampicin, isoniazid, ethambutol and pyrazinamide.

For all cases reported between 2001 and 2005, the outcomes of treatment at 12 months after notification or starting treatment were collated. The definitions for treatment outcome, derived from WHO and European standards, ${ }^{7}$ are summarised in table 1. Data on outcome were only available from 2001 because the national tuberculosis treatment outcome surveillance system was initiated in 2002 for cases reported in 2001. Outcome data for cases reported in 2006 are currently being collated.

\section{Definitions}

For the purposes of this study, cases include all reports of children under the age of 16 years who either have culture confirmed disease due to $M$ tuberculosis complex infection or disease "other than culture confirmed" (a clinician's judgement based on clinical features with or without radiological, histological or tuberculin skin test evidence of tuberculosis, and the decision to treat a patient with a full course of more than two anti-tuberculosis drugs). Pulmonary cases include patients with tuberculosis involving the lungs and/or tracheobronchial tree, with or without a diagnosis of extra-pulmonary tuberculosis, while other sites of disease are classified as extrapulmonary tuberculosis and the specific organ or system affected reported. Ethnic group was classified based on the definitions used by the UK Office of National Statistics (ONS). Multi-drug resistant tuberculosis was defined as resistance to at least rifampicin and isoniazid.

\section{Statistical analysis}

Overall rates per 100000 population, and rates by age, sex and region of reporting, were calculated using the mid-year estimates from the ONS. For place of birth and ethnic group, population estimates from the Labour Force Survey, which have been revised in light of the results of the 2001 census, were used. The statistical significance of the difference between medians was calculated using the $\mathrm{k}$ test for equality of medians. Confidence intervals were calculated using Poisson regression. A p value of 0.05 was considered statistically significant. All analyses were carried out using Intercooled Stata 9 (Stata, College Station, Texas, USA).

\section{RESULTS}

Over the 8-year period, 3563 cases of tuberculosis in children under the age of 16 years were reported, giving an overall

Table 1 Treatment outcome surveillance definitions

\begin{tabular}{|c|c|}
\hline Category & Definition \\
\hline Completion & $\begin{array}{l}\text { Completed full course of therapy within } 1 \text { year of notification/ } \\
\text { starting treatment }\end{array}$ \\
\hline Treatment stopped & $\begin{array}{l}\text { Patient found to have stopped treatment (by himself) or for any } \\
\text { other reason not mentioned below }\end{array}$ \\
\hline Death & $\begin{array}{l}\text { Patients who died while on treatment for tuberculosis, were } \\
\text { diagnosed after death or died without starting treatment }\end{array}$ \\
\hline $\begin{array}{l}\text { Still on treatment } \\
\text { at } 1 \text { year }\end{array}$ & $\begin{array}{l}\text { This includes patients still on treatment at } 1 \text { year because } \\
\text { they are either (i) still on an initially planned course or (ii) had } \\
\text { interruption(s) or the plan was changed due to adverse effects, } \\
\text { drug resistance or non-response }\end{array}$ \\
\hline Lost to follow-up & Patients lost to follow-up before the end of treatment \\
\hline Transferred out & $\begin{array}{l}\text { Responsibility for patients' care transferred to another clinical } \\
\text { team }\end{array}$ \\
\hline Unknown & No treatment details available (eg, lost patient notes) \\
\hline
\end{tabular}

incidence rate of 4.3 per 100000 (95\% CI 4.1 to 4.4) with no significant change $(p=0.27)$ over time.

\section{Demographic characteristics}

The number of cases and disease incidence by demographic characteristics are summarised in table 2 . The median age of all children was 8 years (interquartile range (IOR) 3-13). The highest incidence rate was in the 15-year-old age group at 6.5 per 100000 (95\% CI 5.9 to 7.2) and the lowest rate was in the 5-9-year-old age group (3.2 per $100000,95 \%$ CI 3.0 to 3.4). The rates in all the age groups did not changed significantly over time.

The black African ethnic group had the highest incidence rate at 87.6 per 100000 (95\% CI 82.2 to 93.2), followed by the Pakistani ethnic group at 48.9 per 100000 (95\% CI 45.4 to 52.7). The white population had the lowest rate overall at 1.1 per 100000 (95\% CI 1.0 to 1.2$)$. There has been no significant change between 2001 and 2006 for any of the ethnic groups.

Table 2 Demographic characteristics of paediatric tuberculosis cases in England and Wales, 1999-2006

\begin{tabular}{|c|c|c|c|}
\hline Characteristic & & Number (\%) & $\begin{array}{l}\text { Incidence per } \\
100000(95 \% \mathrm{CI})\end{array}$ \\
\hline \multirow[t]{4}{*}{ Age group (years) } & $0-4$ & $1098(31)$ & 4.5 (4.2 to 4.8$)$ \\
\hline & $5-9$ & $836(24)$ & 3.2 (3.0 to 3.4$)$ \\
\hline & $10-14$ & $1275(36)$ & 4.7 (4.4 to 4.9$)$ \\
\hline & 15 & $354(10)$ & 6.4 (4.6 to 8.5$)$ \\
\hline \multirow[t]{8}{*}{ Ethnicity* } & White (420) & $574(16)$ & $1.1(1.0$ to 1.2$)$ \\
\hline & Black African (828) & $994(28)$ & 87.6 (82.2 to 93.2$)$ \\
\hline & Pakistani (544) & $703(20)$ & $48.9(45.5$ to 52.7$)$ \\
\hline & Indian (262) & $383(11)$ & 29.7 (26.8 to 32.8$)$ \\
\hline & Bangladeshi (120) & $150(4)$ & $24.0(20.3$ to 28.2$)$ \\
\hline & Black Caribbean (75) & $109(3)$ & 14.1 (11.6 to 17.0$)$ \\
\hline & Other & $408(12)$ & \\
\hline & Unknown & $242(7)$ & \\
\hline \multirow[t]{3}{*}{ Gender } & Male & $1717(48)$ & $4.0(3.8$ to 4.2$)$ \\
\hline & Female & $1840(52)$ & 4.5 (4.5 to 4.7$)$ \\
\hline & Missing & $6(0.2)$ & \\
\hline \multirow[t]{3}{*}{ UK born } & Yes & $2037(57)$ & 2.5 (2.4 to 2.7$)$ \\
\hline & No & $1107(31)$ & $37.3(35.2$ to 39.6$)$ \\
\hline & Unknown & $419(12)$ & \\
\hline \multirow[t]{10}{*}{ Region } & East Midlands & $286(8)$ & 4.3 (3.8 to 4.8 ) \\
\hline & East of England & $137(4)$ & 1.6 (1.3 to 1.9$)$ \\
\hline & London & $1543(43)$ & $13.3(12.7$ to 14.0$)$ \\
\hline & North East & $90(3)$ & 2.3 (1.8 to 2.8$)$ \\
\hline & North West & $283(8)$ & $2.6(2.3$ to 2.9$)$ \\
\hline & South East & $173(5)$ & $1.4(1.2$ to 1.6$)$ \\
\hline & South West & $58(2)$ & $0.8(0.6$ to 1.0$)$ \\
\hline & Wales & $104(3)$ & 2.2 (1.8 to 2.7$)$ \\
\hline & West Midlands & $512(14)$ & $5.9(5.4$ to 6.4$)$ \\
\hline & $\begin{array}{l}\text { Yorkshire and the } \\
\text { Humber }\end{array}$ & $377(11)$ & 4.7 (4.2 to 5.2$)$ \\
\hline \multirow{8}{*}{$\begin{array}{l}\text { Year of } \\
\text { notification }\end{array}$} & 1999 & $413(12)$ & 3.9 (3.5 to 4.3$)$ \\
\hline & 2000 & $397(11)$ & $3.8(3.4$ to 4.1$)$ \\
\hline & 2001 & $530(15)$ & $5.0(4.6$ to 5.5$)$ \\
\hline & 2002 & $450(13)$ & 4.3 (3.9 to 4.7$)$ \\
\hline & 2003 & $387(11)$ & 3.7 (3.4 to 4.1$)$ \\
\hline & 2004 & $452(13)$ & $4.4(4.0$ to 4.8$)$ \\
\hline & 2005 & $502(14)$ & $4.9(4.5$ to 5.3$)$ \\
\hline & 2006 & $432(12)$ & 4.2 (3.8 to 4.6$)$ \\
\hline \multicolumn{2}{|l|}{ Overall } & $3563(100)$ & 4.3 (4.1 to 4.4$)$ \\
\hline
\end{tabular}

*Note data are for 2001 to 2006 only (number during that period). Labour Force Survey denominator values prior to 2001 are not comparable due to ethnicity classification change. 
Table 3 Clinical and microbiological characteristics of paediatric tuberculosis cases in England and Wales, 1999-2006

\begin{tabular}{|c|c|c|}
\hline Characteristic & & n (\%) \\
\hline \multirow[t]{3}{*}{ Site of disease } & Pulmonary* & $2120(60)$ \\
\hline & Extra-pulmonary & $1358(38)$ \\
\hline & Unknown & $85(2)$ \\
\hline Diagnostic delay (days) & Median (IQR) & $37(13-89)$ \\
\hline \multirow[t]{3}{*}{ Culture confirmed } & Yes & $948(27)$ \\
\hline & No & $2615(73)$ \\
\hline & Unknown & \\
\hline \multirow[t]{4}{*}{ Species } & $\begin{array}{l}\text { Mycobacterium } \\
\text { tuberculosis }\end{array}$ & $897(25)$ \\
\hline & $M$ bovis & $3(0.1)$ \\
\hline & $M$ africanum & $8(0.2)$ \\
\hline & Unknown & $2669(74)$ \\
\hline \multicolumn{3}{|l|}{ Drug susceptibility } \\
\hline \multirow[t]{2}{*}{ Isoniazid } & Resistant & $78(9.3)$ \\
\hline & Sensitive & $759(90.7)$ \\
\hline \multirow[t]{2}{*}{ Rifampicin } & Resistant & $20(2.4)$ \\
\hline & Sensitive & $817(97.6)$ \\
\hline \multirow[t]{2}{*}{ Ethambutol } & Resistant & $7(0.8)$ \\
\hline & Sensitive & $830(99.2)$ \\
\hline \multirow[t]{2}{*}{ Pyrazinamide } & Resistant & $5(0.6)$ \\
\hline & Sensitive & $830(99.4)$ \\
\hline \multirow[t]{2}{*}{ Multi-drug resistant } & Resistant & $19(2.3)$ \\
\hline & Sensitive & $818(97.7)$ \\
\hline
\end{tabular}

*Includes patients with pulmonary and extra-pulmonary disease. IQR, interquartile range.

Patients born outside the UK (37.3 per 100 000; 95\% CI 28.7 to 39.5$)$ had a tuberculosis rate far higher than those who were born in the UK (2.5 per $100000 ; 95 \%$ CI 2.4 to 2.7). The nonUK born patients were from 85 different countries. The five countries from which the highest number of cases originated were Somalia, Pakistan, India, Zimbabwe and the Philippines. There was a significant increase in the rate in non-UK born children between 1999 and 2006 ( $p=0.007)$. No significant change in rates in UK born children $(p>0.1)$ was observed. For patients born outside the UK, the median duration between arrival in the UK and a diagnosis of tuberculosis was 2 years (IOR 1-4). This varied by ethnicity with a median of 1 year for white, black African and black Caribbean children, 2 years for Pakistani children and 3 years for Indian and Bangladeshi children. The number of cases reported declines with time since arrival in all the ethnic groups.

London had the highest rate of any region (13.3 per 100 000; 95\% CI 12.7 to 14.0). The regional rates have remained relatively constant over this period except in London where there was a trend towards a non-significant increase.

\section{Clinical and microbiological characteristics}

Pulmonary tuberculosis was the commonest presentation (60\%) of the disease (table 3).

The number and proportion of cases by site of disease are summarised in table 4. There was no significant difference in the pulmonary tuberculosis rates between the different age or ethnic groups. Tuberculosis of the central nervous system (CNS) accounted for $2.2 \%$ of cases and was significantly more common in the youngest age group $(0-4$ years $)(p<0.001)$. The median age of those with CNS disease was 5 years (IOR 1-11) compared with 9 years (IOR 3-13) in children without CNS disease $(p=0.001)$.
The median time to diagnosis from onset of symptoms was 37 days (IOR 12-89) (table 3). This ranged from 15 days (IOR 5-50) for those with miliary disease to 90 days (IOR 48-249) for those with bone tuberculosis. Patients who died $(n=13)$ had a median delay between symptom onset and diagnosis of 50 days (IOR 31-207) compared to 33 days (IOR 11-77) among those completing treatment..

Of 948 children with culture confirmed disease, 908 had information on species. Of these, 897 had $M$ tuberculosis, three had $M$ bovis and eight had $M$ africanum. The proportion (number) of culture confirmed cases with rifampicin, isoniazid, pyrazinamide, ethambutol and multi-drug resistant isolates was $2.4 \%$ (20), 9.3\% (78), $0.6 \%$ (5), $0.8 \%$ (7) and $2.3 \%$ (19), respectively.

\section{Treatment outcomes}

The majority of reported cases (88\%) completed treatment at 12 months. This proportion remained stable at between $88 \%$ and $90 \%$ during the study period (table 5). The proportion completing treatment was, however, lower among children with severe forms of disease (miliary tuberculosis: 68\%; meningitis: 60\%). Overall, 13 children (0.7\%) died and 59 $(3.1 \%)$ were lost to follow-up during this period.

\section{DISCUSSION \\ Main findings}

The rate of tuberculosis in children in England and Wales did not change significantly between 1999 and 2006 despite the continuing increase in the adult population. Rates of disease among children are also comparable to those reported from the previous 1993 and 1998 surveys. ${ }^{5}$ The overall rate, however, masks different patterns in some subpopulations. The rate is increasing in non-UK born children. Also, the bulk of the disease burden falls on non-UK born children, especially those born in Africa, and those of black African, Indian, Pakistani and Bangladeshi ethnic origin.

Pulmonary tuberculosis was the commonest clinical presentation of childhood disease, with no significant variation between the different age and ethnic groups. While the overall proportion of CNS tuberculosis was low, there was an increase in CNS disease in younger children less than 4 years of age. Diagnosis was generally prompt. Nevertheless, some patients, such as those with tuberculosis of the bone, experienced long delays. Culture confirmation rates remain low and the proportion of cases with rifampicin, isoniazid and multi-drug resistance was high compared to that reported in adults, ${ }^{4}$ although the total numbers were much smaller. Nearly $90 \%$ of patients completed treatment and only a small proportion were lost to follow-up or died.

\section{Strengths and limitations}

This study presents the most recent national data available on the demographic, clinical and microbiological patterns of tuberculosis in children. Although other studies have reported trends in tuberculosis, they are too limited by the geographical area covered and the number of cases to allow detailed analysis of features. ${ }^{5} 8$

This study relied on surveillance data and, therefore, does not include other important clinical data such as BCG vaccination status, whether cases are part of an outbreak and HIV status of the children. Despite the use of national data over an 8-year period, subgroup analyses were limited by small numbers. Furthermore, the matching of various data sources inevitably 
Table 4 Paediatric tuberculosis disease sites by age and ethnicity, England and Wales, 1999-2006

\begin{tabular}{|c|c|c|c|c|c|c|c|c|c|c|c|}
\hline Characteristic & $\begin{array}{l}\text { Pulmonary, } \\
\text { n (\%) }\end{array}$ & $\begin{array}{l}\text { Pleural, } \\
\text { n (\%) }\end{array}$ & $\begin{array}{l}\text { Intra-thoracic } \\
\text { lymph, nodes } \\
\text { n (\%) }\end{array}$ & $\begin{array}{l}\text { Lymph } \\
\text { nodes, } \\
\text { n (\%) }\end{array}$ & $\begin{array}{l}\text { Bone, } \\
\text { n (\%) }\end{array}$ & $\begin{array}{l}\text { Central } \\
\text { nervous } \\
\text { system, } \\
\text { n (\%) }\end{array}$ & $\begin{array}{l}\text { Gastro- } \\
\text { intest-inal, } \\
\text { n (\%) }\end{array}$ & Miliary* & $\begin{array}{l}\text { Other } \\
\text { sites } \dagger \text {, } \\
\text { n (\%) }\end{array}$ & $\begin{array}{l}\text { Un- } \\
\text { known, } \\
\text { n (\%) }\end{array}$ & $\begin{array}{l}\text { Total, } \\
\text { n (\%) }\end{array}$ \\
\hline $0-4$ & $681(62.0)$ & $14(1.3)$ & $138(12.6)$ & $110(10.0)$ & $15(1.4)$ & $34(3.1)$ & $3(0.3)$ & $16(1.5)$ & $74(6.7)$ & $13(1.2)$ & $1098(100)$ \\
\hline $5-9$ & 487 (58.3) & $17(2)$ & $151(18.1)$ & $81(9.7)$ & $19(2.3)$ & $19(2.3)$ & $7(0.8)$ & $2(0.2)$ & $50(6)$ & $3(0.4)$ & $836(100)$ \\
\hline $10-14$ & 738 (57.9) & $42(3.3)$ & $199(15.6)$ & $137(10.8)$ & $26(2.0)$ & $19(1.5)$ & $22(1.7)$ & $11(0.9)$ & $76(5.7)$ & $5(0.4)$ & $1275(100)$ \\
\hline White & $347(60.5)$ & $10(1.7)$ & $61(10.6)$ & 83 (14.5) & $13(2.3)$ & $14(2.4)$ & 0 & $4(0.7)$ & $39(6.9)$ & $3(0.5)$ & $574(100)$ \\
\hline Black Caribbean & 64 (58.7) & $3(2.8)$ & $20(18.4)$ & $9(8.3)$ & $2(1.8)$ & $3(2.8)$ & $2(1.8)$ & $2(1.8)$ & $4(3.7)$ & $0(0)$ & $109(100)$ \\
\hline Black African & $636(64)$ & $19(1.9)$ & $122(12.3)$ & $92(9.3)$ & $16(1.6)$ & $23(2.3)$ & $16(1.6)$ & $7(0.7)$ & $63(6.3)$ & $0(0)$ & $994(100)$ \\
\hline Indian & $206(53.8)$ & $17(4.4)$ & $59(15.4)$ & $46(12)$ & $11(2.9)$ & $5(1.3)$ & $4(1)$ & $4(1.0)$ & $28(7.3)$ & $3(0.8)$ & $383(100)$ \\
\hline Pakistani & $395(56.2)$ & $12(1.7)$ & $148(21.1)$ & $64(9.1)$ & $16(2.3)$ & $14(2)$ & $10(1.4)$ & $5(0.7)$ & $35(5)$ & $4(0.6)$ & $703(100)$ \\
\hline Bangladeshi & $98(65.3)$ & $1(0.7)$ & $18(12)$ & 17 (11.3) & $2(1.3)$ & $3(2)$ & 0 & 0 & $10(6.8)$ & $1(0.7)$ & $150(100)$ \\
\hline
\end{tabular}

${ }^{*} \mathrm{~A}$ further 32 patients were coded as miliary and pulmonary; †genitourinary, laryngeal, skin and other.

means that there will be some errors. This could potentially lead to non-differential misclassification and, therefore, its effect will be to reduce the ability to detect associations. The matching algorithm has been used for over 8 years and has been validated through detailed audits in a number of regions.

The Labour Force Survey, which was used to calculate rates by ethnic groups and place of birth, is based on a population sample, and thus estimates are liable to sampling errors, particularly for smaller population subgroups. Rates by ethnic group and place of birth should therefore be interpreted with caution.

A recent study by the British Paediatric Surveillance Unit (BPSU) suggests undernotification may be as high as $20 \%$, therefore potentially underestimating the burden of disease particularly in children under 5 years of age. ${ }^{9}$ More childhood tuberculosis cases were, however, reported to the ETS system compared to the BPSU survey, suggesting that the undernotification observed could be due, at least in part, to failures in matching the two databases. This study, however, concluded that the ETS system provides a representative picture of the demographics of tuberculosis in children in the UK.

\section{Interpreting the findings}

Tuberculosis among children remains largely a disease of those born to parents belonging to an ethnic minority. In contrast to the fact that about $72 \%$ of all tuberculosis patients are reported to have been born outside the UK, ${ }^{4}$ only $30 \%$ of paediatric tuberculosis patients were non-UK born. The results of this study support those of previous reports regarding the higher risk of tuberculosis among black African children compared to other ethnic groups. ${ }^{568}$ Although stable, the rates in Pakistani children remain high. The increasing rate of tuberculosis in children born outside the UK probably reflects the changing demographics of immigration to the UK, with more immigrants coming from countries with a high incidence of tuberculosis. The static, rather than declining, rates in the 0-4-year-age group is a particular cause for concern as it is a marker of ongoing transmission rather than reactivation of old disease. ${ }^{1}$

The diagnosis of tuberculosis in children remains problematic. Only $27 \%$ of paediatric cases had a positive microbiological diagnosis compared to $60 \%$ of all tuberculosis cases. ${ }^{4}$ Potential explanations for this low rate of confirmation may include failure to report a proportion of culture confirmed cases, inadequacies in the data linkage algorithms used, different spectrums of disease or difficulties in obtaining specimens, especially in smaller children and infants. The lower confirmation rate could also be related to less aggressive investigation in paediatric cases diagnosed following an adult index case. It would be useful for the ETS system in the future to collect information on whether childhood tuberculosis cases were identified following contact investigation. Even in the best of circumstances, culture confirmation rates in children are usually low. ${ }^{10}$ Further research to improve the diagnosis of tuberculosis in children is needed.

The proportion of children completing treatment, in contrast, is very high, exceeds the WHO recommendation of $85 \%$ and is well above the overall (adult and paediatric) rate of $79 \%{ }^{4}$ As expected, treatment completion rates were lower among those

Table 5 Treatment outcomes reported by year, England and Wales, 2001-2005

\begin{tabular}{|c|c|c|c|c|c|c|}
\hline Treatment outcome & $\begin{array}{l}2001 \\
\text { n (\%) }\end{array}$ & $\begin{array}{l}2002 \\
\text { n (\%) }\end{array}$ & $\begin{array}{l}2003 \\
\text { n (\%) }\end{array}$ & $\begin{array}{l}2004 \\
\text { n }(\%)\end{array}$ & $\begin{array}{l}2005 \\
\text { n (\%) }\end{array}$ & $\begin{array}{l}\text { Total, } \\
\text { n (\%) }\end{array}$ \\
\hline Treatment completed & $336(90)$ & $338(89)$ & $300(87)$ & $347(88)$ & $395(88)$ & $1729(88)$ \\
\hline Died & $2(0.5)$ & $4(1.1)$ & $2(0.6)$ & $3(0.8)$ & $2(0.5)$ & $13(0.7)$ \\
\hline Still on treatment & $7(1.9)$ & $9(2.4)$ & $12(3.5)$ & $15(3.8)$ & $20(4.5)$ & $63(3.3)$ \\
\hline Treatment stopped & $3(0.8)$ & $2(0.5)$ & $3(0.9)$ & $7(1.8)$ & $1(0.2)$ & $16(0.8)$ \\
\hline Transferred out & $6(1.6)$ & $4(1.1)$ & $11(3.2)$ & $12(3)$ & $10(2.3)$ & $43(2.2)$ \\
\hline
\end{tabular}


with more severe forms of disease, such as CNS tuberculosis which would require a longer duration of treatment. About 3\% of children were still on treatment at 12 months. These were mainly children with either drug resistant tuberculosis or tuberculosis meningitis. Unfortunately, a very small number of children still die of tuberculosis or are lost to follow-up. A detailed clinical audit of these cases will provide further insight and support the management of such cases.

\section{Clinical and public health implications}

The higher incidence of childhood tuberculosis among certain ethnic minority and age groups supports the current policy of selective BCG vaccination. ${ }^{11}$ However, the higher incidence of tuberculosis, particularly severe forms of tuberculosis such as CNS disease, in younger age groups may raise questions regarding the effectiveness of the BCG vaccination programme. Rates among UK born white children remain very low. It is also unlikely that new entrant screening for tuberculosis disease can prevent these cases. As only $30 \%$ of patients are non-UK born and many of these develop disease after arrival in the UK, screening for tuberculosis disease at port of entry is unlikely to make any impact on $70 \%$ of cases.

Although we did not collect information on whether cases were linked to non-household outbreaks, it appears that the contribution of transmission in institutional settings is probably limited in view of the concentration of cases in ethnic minority groups. This, however, can not be excluded and further investigation is required.

In view of the diagnostic delays observed and the adverse outcome of care in some cases, clinicians should have a higher index of suspicion for tuberculosis. Reducing delay is important not only to improve clinical outcome but also because extensive transmission from children has been reported. ${ }^{12}{ }^{13}$ Appropriate management models suggested in national guidelines should also be followed. ${ }^{14} 15$

The relatively high rates of drug resistance identified demonstrates that drug resistance is not an issue limited to adults. Paediatricians managing children with tuberculosis should have a higher index of suspicion for resistance and recommended multi-drug therapy should always be used. ${ }^{15}$

\section{CONCLUSION}

The overall rate of tuberculosis in children has remained stable, a high proportion of patients complete treatment and mortality remains low. Unfortunately, rates of tuberculosis are very high and rising in non-UK born children. While the mortality rates are low, 13 children still died during the study period. A confidential enquiry into these deaths would be useful to inform management of childhood tuberculosis.

Acknowledgements: We are grateful to David Quinn for record linkage and to all clinicians and microbiologists who contribute data to the national surveillance system. We wish to thank Michelle Kruijshaar for comments on the manuscript.

\section{Competing interests: None.}

Ethics approval: This study was carried out with national surveillance data. The Health Protection Agency has PIAG approval to hold and analyse national surveillance data for public health purposes.

Contributions: All authors have seen and approved the final manuscript. In addition, Matthew Laundy and Ibrahim Abubakar conducted the analysis. All authors contributed to writing the paper. Dr Ibrahim Abubakar is guarantor.

\section{REFERENCES}

1. World Health Organization. Guidance for national programmes on the management of tuberculosis in children. WHO/HTM/TB/2006.371. Geneva: WHO, 2006.

2. Stop TB Partnership Childhood TB Subgroup, World Health Organization. Guidance for national tuberculosis programmes on the management of tuberculosis in children. Chapter 1: introduction and diagnosis of tuberculosis in children. Int J Tuberc Lung Dis 2006;10:1091-7.

3. Stop TB Partnership Childhood TB Subgroup, World Health Organization. Guidance for national tuberculosis programmes on the management of tuberculosis in children. Chapter 2: anti-tuberculosis treatment in children. Int J Tuberc Lung Dis 2006;10:1205-11.

4. Kruijshaar ME, French CE, Anderson C, et al. Tuberculosis in the UK: annual report on tuberculosis surveillance and control in the UK. London: Health Protection Agency, 2007.

5. Balasegaram S, Watson JM, Rose AM, et al. A decade of change: tuberculosis in England and Wales 1988-98. Arch Dis Child 2003;88:772-7.

6. Fathoala B, Evans MR, Campbell IA, et al. Active surveillance for tuberculosis in Wales: 1996-2003. Arch Dis Child 2006;91:900-4.

7. Veen J, Raviglione M, Rieder HL, et al. Standardized tuberculosis treatment outcome monitoring in Europe. Recommendations of a Working Group of the World Health Organization (WHO) and the European Region of the International Union Against Tuberculosis and Lung Disease (IUATLD) for uniform reporting by cohort analysis of treatment outcome in tuberculosis patients. Eur Respir J 1998;12:505-10.

8. Atkinson $\mathbf{P}$, Taylor $\mathrm{H}$, Sharland $\mathrm{M}$, et al. Resurgence of paediatric tuberculosis in London. Arch Dis Child 2002;86:264-5.

9. Teo SS, Alfaham M, Evans MR, et al for the British Paediatric Surveillance Unit Childhood Tuberculosis study group. Epidemiology of childhood tuberculosis in the United Kingdom and Republic of Ireland. Arch Dis Child. In press.

10. Eamranond $\mathbf{P}$, Jaramillo E. Tuberculosis in children: reassessing the need for improved diagnosis in global control strategies. Int J Tuberc Lung Dis 2001:5:594-603.

11. Joint Committee on Vaccination and Immunisation. BCG statement. Available from http://www.advisorybodies.doh.gov.uk/jcvi/bcg-jcvi-statement_7june2007.pdf (accessed 28 August 2008).

12. Howard J, Paranjothy S, Thomas $\mathrm{S}$, et al. Outbreak of tuberculosis in a junior school in south-eastern England. Euro Surveill 2007;12:E070628.1.

13. Curtis AB, Ridzon R, Vogel $R$, et al. Extensive transmission of Mycobacterium tuberculosis from a child. N Engl J Med 1999;341:1491-5.

14. Adalat S, Paliwalla M, Novelli V, et al. A survey of tuberculosis services in the UK Arch Dis Child 2008;93:575-77.

15. National Collaborating Centre for Chronic Conditions. Tuberculosis: clinical diagnosis and management of tuberculosis, and measures for its prevention and control. London: Royal College of Physicians, 2006. 